\title{
PARTICLE DEPOSITION STUDY IN A HORIZONTAL SQUARE CHANNEL USING HIGH-RESOLUTION IMAGING
}

\author{
D. Orea ${ }^{1}$, R. Chavez ${ }^{1}$, D.T. Nguyen ${ }^{1}$, R. Vaghetto ${ }^{1}$, N. K. Anand ${ }^{1}$, Y. A. Hassan ${ }^{1}$, \\ and $P$. Sabharwall ${ }^{2}$ \\ ${ }^{1}$ Texas A\&M University \\ College Station, TX, 77843 \\ ${ }^{2}$ Idaho National Laboratory \\ Idaho National Laboratory, Idaho Falls, ID, 83415 \\ oreadaniel@tamu.edu, reyjrchavez23@tamu.edu, thien.duy.ng@tamu.edu, \\ r.vaghetto@tamu.edu,nkanand@tamu.edu, y-hassan@tamu.edu,piyush.sabharwall@inl.gov
}

\begin{abstract}
The ongoing research at Texas A\&M University (TAMU) aims to develop and implement measurement techniques to quantify fission product transport and related phenomena in a GasCooled Fast Reactor (GCFR). This study summarizes the experimental measurements performed in a Proof-of-Concept Facility (PCF) to understand particle transport under various Reynolds numbers. Using high-resolution high-magnification optical measurement techniques, surrogate particle deposition was measured on the bottom wall surface of the PCF. The particle deposition over time was observed to decrease as the Reynolds number increased. In addition, the particle spatial distributions increased approaching the side wall of the PCF. This state-ofthe art optical technique allows for the non-intrusive measurements of micron-sized particles near the wall surface.
\end{abstract}

KEYWORDS: Particles Deposition, Non-Intrusive Optical Measurements

\section{INTRODUCTION}

Texas A\&M University (TAMU), led by Idaho National Laboratory, is participating in the U.S. Department of Energy, Office of Nuclear Energy's Versatile Test Reactor (VTR) program to develop instrumentation and tools for a proposed fast spectrum test reactor. The research being performed at TAMU aims to develop and implement measurement techniques to quantify fission product transport and deposition. These fission products may be released into the primary system of a Gas-Cooled Fast Reactor (GCFR) and potentially the reactor confinement building. Previous research conducted on GCFR's discuss the potential risk of particle deposition within the reactor building. These particles have shown to have an affinity towards fission products, making the dust a potential source term. The particles can range from submicron to an order of $10 \mu \mathrm{m}$ [1]. Techniques developed to investigate transport of particles will be ultimately tested in a downscaled GCFR experimental facility. Prior to applying the measurement techniques to the downscaled experimental facility, it is important to verify and validate the techniques and their associated uncertainties. This is accomplished by conducting preliminary tests in a horizontal square channel referred to as the Proofof-Concept Facility (PCF). 
This paper will discuss the experimental study of surrogate particle deposition in a PCF, which has a 7.62 $\mathrm{cm}^{2}$ cross-sectional area. The PCF channel has three parts, each $60.96 \mathrm{~cm}$ in length for a combined total length of $182.88 \mathrm{~cm}$. The first part allows for the flow development, while the second part of the channel is used to implement the developed techniques, and the third part is used for particle filtration. The consideration of surrogate particles to study the aerodynamics behaviors of fission particles is based on the dynamic similarity of Stokes and Reynolds numbers. Depending on the fluid Reynolds numbers and the particle Stokes numbers, particulates released into the PCF have their channel wall depositions affected by gravitational settling, turbulent deposition, and other forces [2].

\section{DESCRIPTION OF THE PROOF-OF-COONCEPT TEST FACILTIY}

In order to study the fluid behavior and particle deposition on the surface of a material, a Proof-of-Concept Facility (PCF) was constructed to facilitate experiments. The PCF, shown in Fig 1, has a $7.62 \mathrm{~cm}^{2} \mathrm{cross}^{-}$ sectional area and is a total of $182.88 \mathrm{~cm}$ is length. The PCF is divided into three equal sections of $60.96 \mathrm{~cm}$ in length. The first section of the facility allows for flow development. The third is used for particle filtration and prevents backflow. The middle section (i.e. test section) is used to measure parameters of interest such as the fluid velocity and particle deposition.

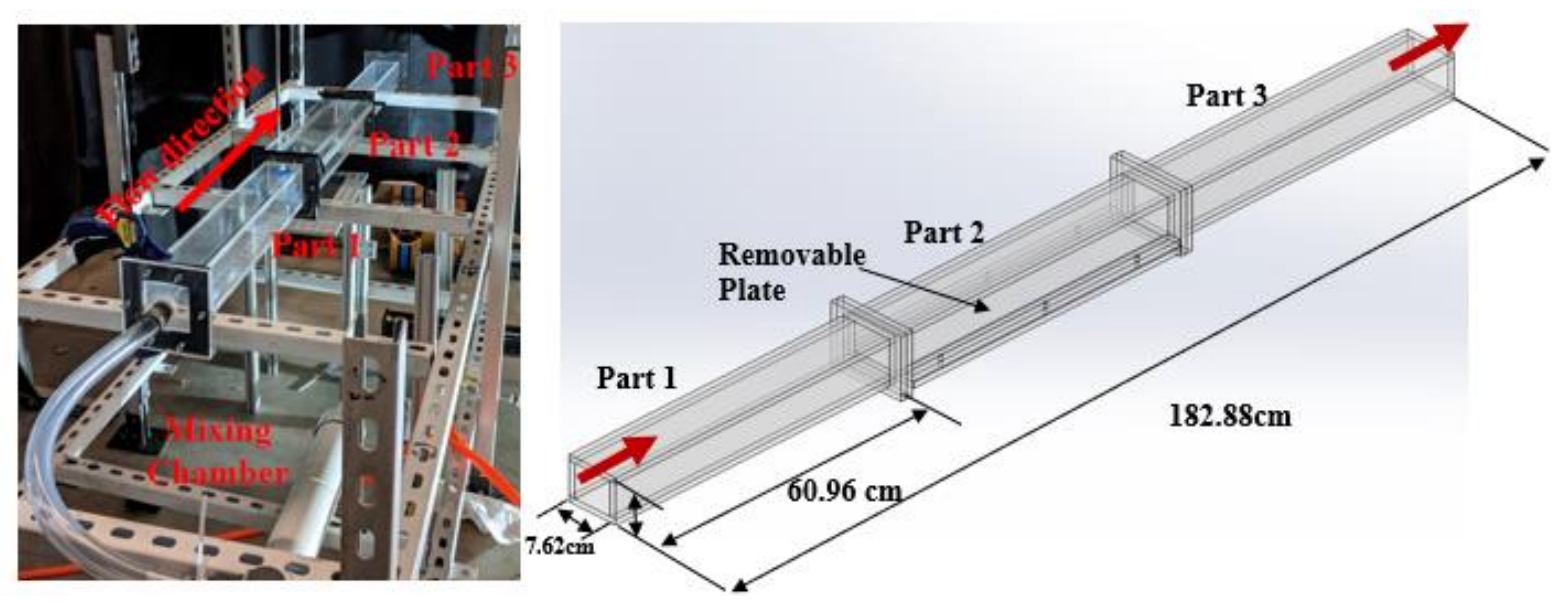

Figure 1. Test channel and Computer-Assisted Drawing (CAD) of the PCF.

This middle test section is constructed to allow the removal of a single wall. This feature is important as it allows the surface of the test section to be modified and accommodated for a wide range of experiments. The facility is connected to an air compressor with capabilities to adjust the air-flow rates. The operating fluid is air passing through a mixing chamber where the fluid-particle mixture can be created prior to entering the inlet of the test facility. A hot-wire anemometer is used to measure the test section inlet velocity and to determine the volumetric flow rate of the mixture. In this study, polypropylene microspheres are used as surrogate particles with a density of $42 \mathrm{~kg} / \mathrm{m}^{3}$. The mean particle size, $d_{\text {part }, m}$, ranges from $25-$ $50 \mu \mathrm{m}$. The microspheres yield a Stoke number $S t k_{\text {part }} \ll 1$.

\subsection{Scaling of Surrogate Particle}

The following non-dimensional numbers were applied to model the physics of a prototypical reactor and to determine the physical properties of the working fluid along with the particle size and density. Reynolds 
number, $R e$, will account for the similarity between the flow conditions of the prototype and model. $R e$ is defined as,

$$
R e_{f}=\frac{U_{f} \rho_{f} L}{\mu_{f}}
$$

where $\mathrm{L}$ is the characteristic length, $U_{f}$ is the fluid velocity, $\rho_{f}$ is the fluid density, and $\mu_{f}$ is the fluid dynamic viscosity. To characterize the particle behavior in the fluid, Stokes number, Stk, is considered. Stk is defined as,

$$
S t k=\frac{\rho_{\text {part }} d_{\text {part }}^{2}}{18 \mu_{f}} \frac{U_{f}}{L}
$$

where "part" indicates particle, $\rho_{\text {part }}$ represents the particle density, and $d_{\text {part }}$ is the particle diameter. Note that the current experiments will be performed under isothermal conditions. To guarantee thermalfluid dynamic similarity between prototype and model, the ratio of the dimensionless number is set to unity. From Reynolds similarity, the scaling of fluid velocity can be algebraically solved in terms of fluid properties and the prototype fluid flow. It is expressed as,

$$
U_{f, m}=\left(\frac{\rho_{f, P}}{\rho_{f, m}} \cdot \frac{\mu_{f, m}}{\mu_{f, P}} \cdot f_{\text {scale }}\right) U_{f, p}=C_{U} \cdot U_{f, p}
$$

where $C_{U}$ is the fluid velocity coefficient that is determined based on fluid properties and $f_{\text {scale }}$ is a geometric scaling factor. Note that $f_{\text {scale }}$ can be adjusted to adequately model the physics of a scaled experimental facility. Similarly, from the Stoke relation, the scaling of particle diameter can be determined based on fluid and particle properties as expressed below,

$$
d_{\text {part }, m}=\left(\frac{1}{f_{\text {scale }}} \sqrt{\frac{\rho_{\text {part }, p}}{\rho_{\text {part }, m}} \frac{\rho_{f, m}}{\rho_{f, p}}}\right) d_{\text {part }, p}=C_{d p} \cdot d_{\text {part }, p}
$$

where $C_{d p}$ is a particle diameter coefficient determined based on fluid properties and geometric scale. From Eq. 3 and Eq. 4, the working fluid velocity and surrogate particle size can be modified within the model based on the prototype being investigated. Particle deposition onto the channel walls is affected by gravitational and turbulent deposition, which are also dependent on the fluid Reynolds number and the particle Stokes number. For a full description of the scaling approach refer to [3, 4].

\section{EXPERIMENTAL APPROACH FOR PARTICLE DEPOSITION MEASUREMENTS}

The PCF can perform a wide range experiments using state-of-the-art measurement techniques. As shown in Fig. 2, the PCF is capable of taking measurements at a variety of locations within the test section. 


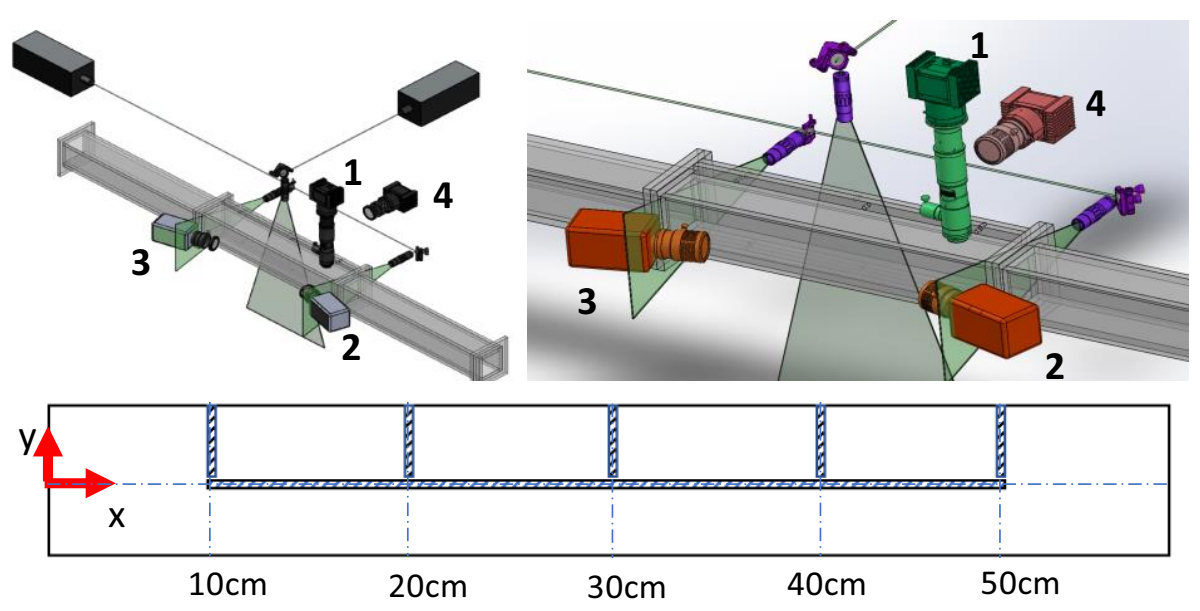

Figure 2. (Top-Left \& Top-Right) Available Measurement Locations in PCF; (Bottom) Measurement Scan Locations for Particle Distribution Using Camera 1 along the Bottom Wall

In this study, camera 1 is an 8 Megapixel camera with a maximum resolution of 2,458 $\times 3,312$ pixels with a pixel size of $5.5 \times 5.5 \mu \mathrm{m}^{2}$. The high-resolution camera is adapted with a $12 \mathrm{x}$ zoom $(0.58-7 \mathrm{x}$ magnification range) lens to measure particles on the test section bottom wall. A high-power light source is placed under the channel to illuminate the entire test section. For the test performed in this study, the camera 1 operated at $8 \mathrm{~Hz}$ and $0.06 \mathrm{~Hz}$. The higher frequency is used to collect images while the fluid is flowing through the $\mathrm{PCF}$, while the lower frequency is applied to capture images once flow is stopped.

There were two experimental measurements performed during the image capturing. The first measurement consisted of capturing images of the particles depositing on the bottom wall approximately $10 \mathrm{~cm}$ from the inlet of the test section. This condition provided the particle deposition on the bottom wall as a function of the time. The second measurement captured images of the axial and lateral particle distribution shown in Fig 2. Depending on the condition, sequences of 40 to 180 images were collected and post-processed using in-house MATLAB codes and ImageJ $[5,6]$.

\section{EXPERIMENTAL RESULTS}

Results corresponding to a ten set of various Reynolds numbers; namely, $\operatorname{Re}_{1}=750, \operatorname{Re}_{2}=1,750, \operatorname{Re}_{3}=$ $2,750, R_{4}=3,000 \operatorname{Re}_{5}=3,500, R_{6}=4,000, R_{7}=4,500, R_{8}=5,000, R_{9}=6,250, R_{10}=7,000$ are presented for the measurement of particle deposition over time. For the measurements of the axial and lateral particle distributions, $\mathrm{Re}_{5}, \mathrm{Re}_{8}$, and $\mathrm{Re}_{9}$ are presented. The raw images capture were converted into binary images by applying an intensity threshold. From the resulted binary images, all particles were counted by assuming a particle presence when the image intensity was at a maximum and vice versa. The set images shown in Fig. 3 demonstrate the two-step process from the original image captured. 


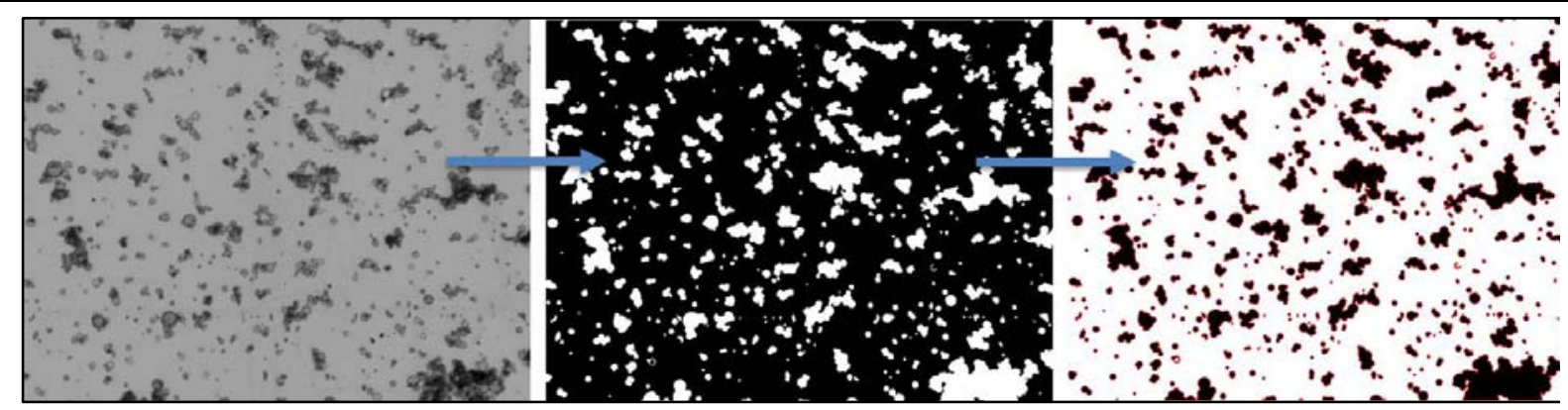

Figure 3. (Left) Raw Image; (Middle) Binary Image; (Right) Counted Particles.

\subsection{Results of Particle Deposition over Time}

The particle deposition was measured within a duration of 21.375 seconds. During this interval, images at the bottom wall were acquired to estimate the particle deposition over time. The number of particles per image is estimated by calculating the projected area of the particle clusters. Using the information of area of the particle clusters, seen in the left image Fig. 3, the number of particles that can occupy the area is estimated using the average dimeter of the particles.

$$
N_{\text {part }} \cong \frac{A_{\text {cluster }}}{A_{\text {part }, \text { mean }}}
$$

where $N_{\text {part }}$ is the number of particles, $A_{\text {cluster }}$ is the area of the cluster after the image processing, and $A_{\text {part, mean }}$ is the mean area of a particle. The normalized particle deposition as a function of time with a $2^{\text {nd }}$ degree polynomial fit is shown in Fig. 4.

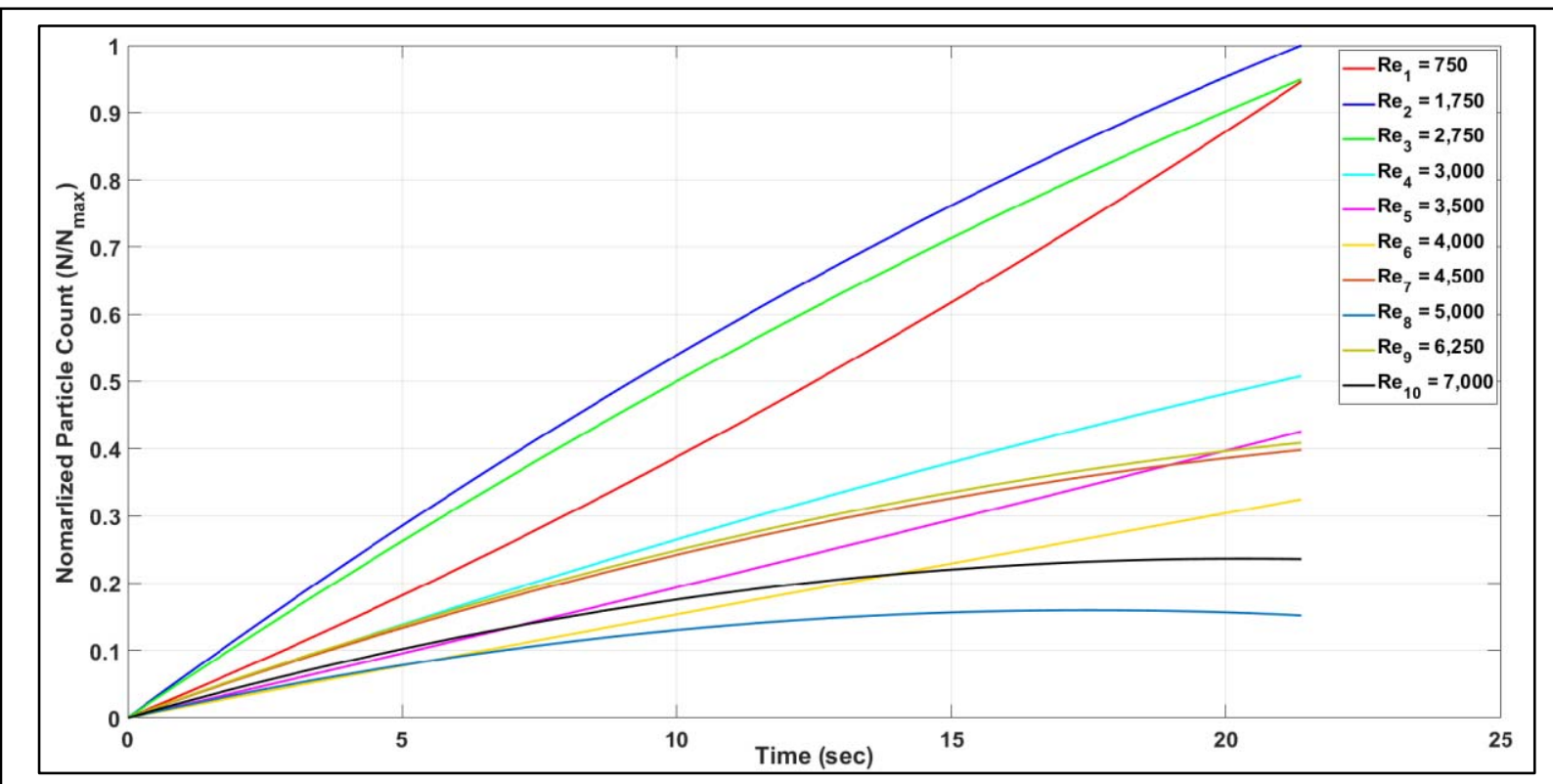

Figure 4. Normalized Particle Count Over Time for 10 Reynolds Numbers

The particle deposition on the wall surface of the PCF decreased as the Reynolds number increased. The decreasing deposition indicates that the particle penetration is increasing as the 
Reynolds number increase. However, there is a separation between $\leq \mathrm{Re}_{3}$. When the Reynolds exceeds $\mathrm{Re}_{3}$, the deposition decreases by approximately $40 \%$. This effect can be seen in Figure 4 . This is mostly caused by the random motion of the fluid as it transitions to turbulent flow.

\subsection{Results of Particle Spatial Distribution}

To acquire the spatial distributions of particles along the bottom wall of the PCF, experimental images were taken along different axial and lateral locations shown in blue in Fig 2. A similar approach was applied and the concentration of the particles per unit area was calculated,

$$
C_{\text {part }} \cong \frac{A_{\text {part }}}{A_{\text {step }}}
$$

where $A_{\text {part }}$ is the area occupied by particles and $A_{\text {step }}$ is the area of the image taken.

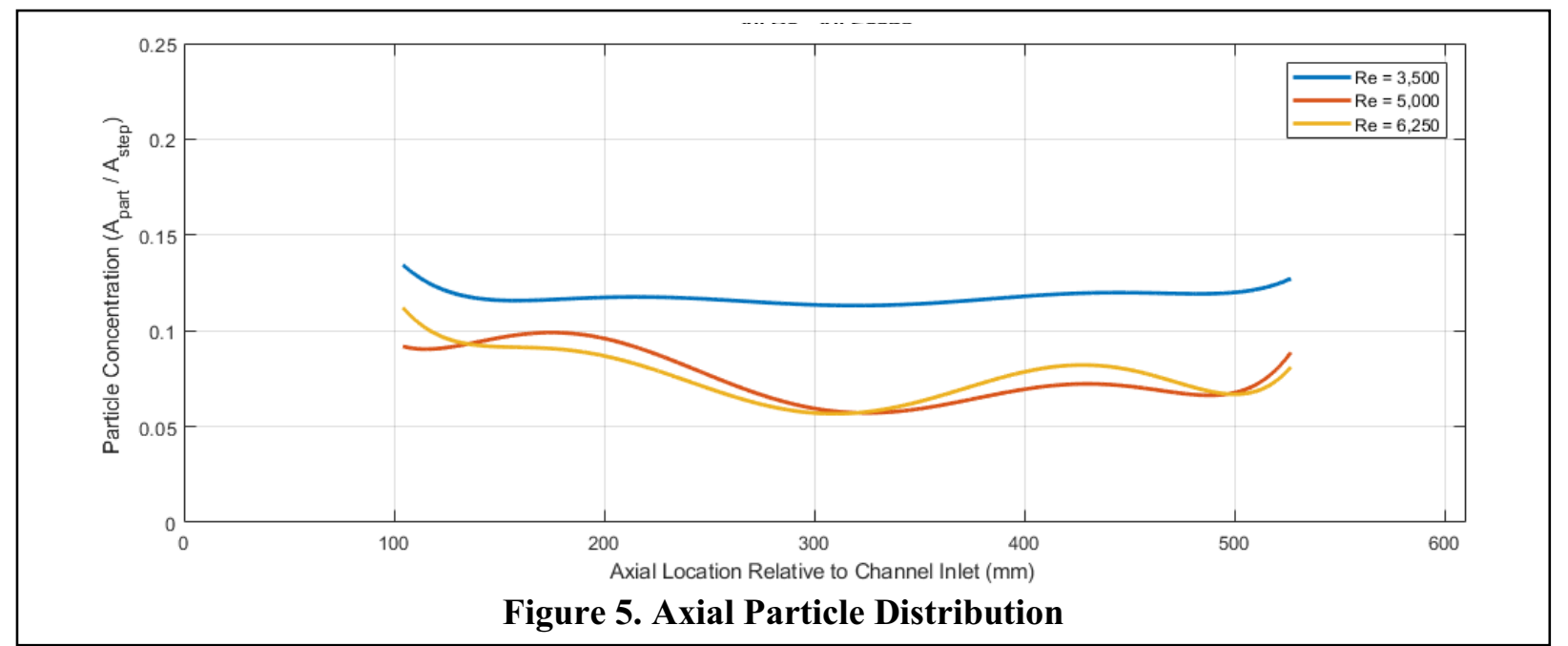

The profile shown in Fig. 5 represent the axial distributions beginning approximately $100 \mathrm{~mm}$ from the inlet of the test section. A key takeaway from the axial particle distribution results are the effects of turbulence of deposition. From the three Reynolds presented, $R e=3,500$ has the highest particle deposition. The other two Reynolds numbers shown a decreased deposition. This is in agreement with the results shown in Figure 4. The highest two Reynolds number also depict the random nature of the fluid flow.
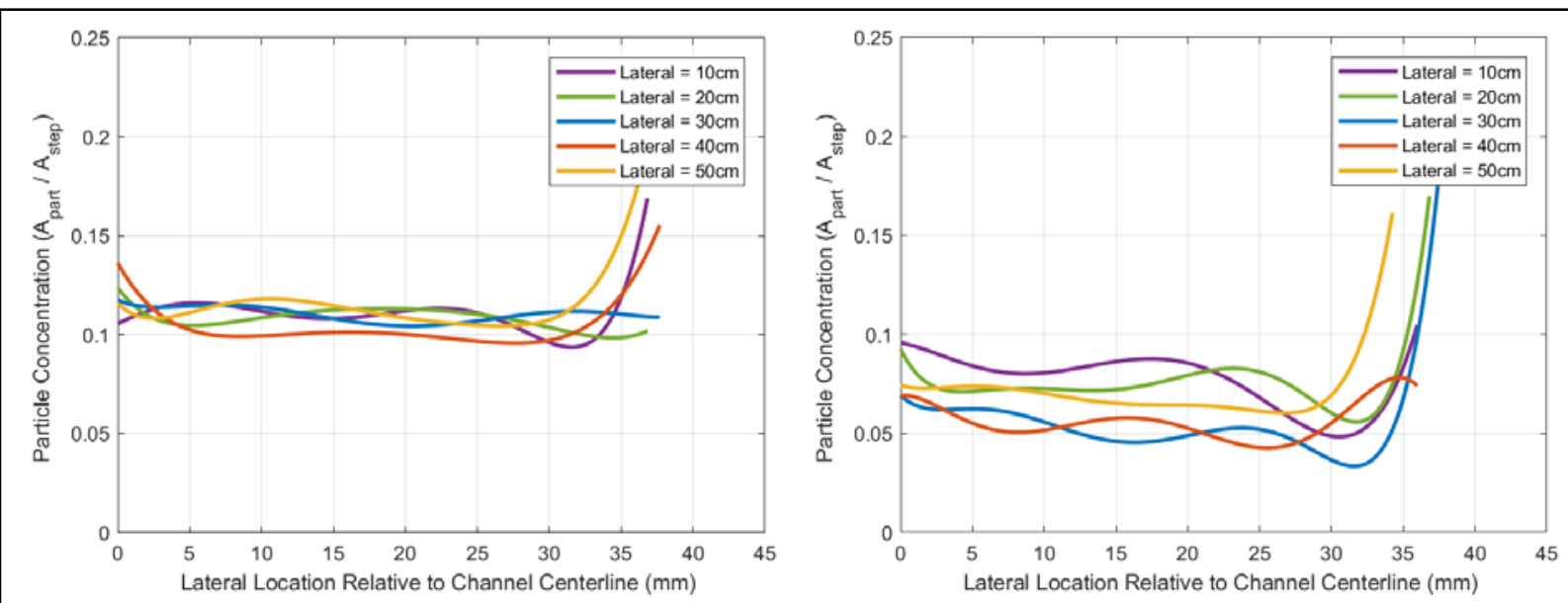

Figure 6. Lateral Particle Distribution (Left) $\operatorname{Re}_{3}=\mathbf{3 , 5 0 0}$ (Right) $\operatorname{Re}_{4}=\mathbf{5 , 0 0 0}$ 
The left and right graphs in Fig. 6 represent the lateral distribution starting from the centerline of the test section, as depicted in blue in Fig. 2. The lateral centerline on the bottom surface wall of the test section is represented at $\mathrm{y}=0 \mathrm{~mm}$ and the side wall boundary is represented at $\mathrm{y}=38.1 \mathrm{~mm}$ in Fig. 6 . The resulting lateral profiles at different locations downstream of the inlet had a similar trend of increasing deposition near the side wall of the PCF. This agrees with numerical simulations of particle deposition in turbulent square channels [7].

\section{SUMMARY}

This paper summarizes the experimental study of surrogate particle deposition in a PCF to take a step further in understanding the physical phenomena in the particle transport and fission products in a GCFR. The scaling approach adopted maintains the similarities of Reynolds and Stokes numbers, which allows the use of a variety of surrogate particles and working fluids based on the prototype being scaled.

The experimental measurements performed in the PCF used polypropylene microspheres as the surrogate particle for gas-cooled reactor dust. The particle deposition over time on the wall surface and the axial and spatial distributions were acquired using high-resolution, high-magnification imaging techniques. It was observed that the particle deposition over time based on the Reynolds number agrees with the hypothetical particle behavior. That is, at low Reynolds numbers gravitational settling is a potential dominant force while at high Reynolds turbophoresis becomes dominant. This is observed in Fig. 4 were $\operatorname{Re}_{1}, \operatorname{Re}_{2}$, and $\operatorname{Re}_{3}$ have higher particle deposition. For the greater Reynolds number, $\mathrm{Re}_{4}$, the particle deposition decreases compared to the lower Reynolds numbers. The axial distributions shown in Fig. 5 demonstrate that the particle deposition slightly increases near the inlet and outlet of the test section. The lateral distribution shown in Fig. 6 demonstrate an increase in particle deposition near the side wall of the PCF.

Future work will be performed to study the particle penetration in the PCF and the effects of a modified the surface with a different material. In addition, experimental results will be compared to numerical simulations. Understanding the surrogate particle transport in the PCF will aid in further understanding the fission product gases in a GCFR.

$\begin{array}{ll}\text { A } & \text { Area } \\ C_{d p} & \text { Coefficient of Particle Diameter } \\ C_{U} & \text { Coefficient of Fluid Velocity } \\ C_{\text {part }} & \text { Area Occupied By Particles per Unit Area } \\ d_{\text {part }, m} & \text { Particle Diameter of Experimental Facility Model } \\ d_{\text {part }, p} & \text { Particle Diameter of Reactor Building Prototype } \\ f_{\text {scale }} & \text { Geometric Scaling Factor } \\ \mathrm{L} & \text { Characteristic Length } \\ \mathrm{N}_{\text {part }} & \text { Number of Particles } \\ \mu & \text { Dynamic Viscosity } \\ v & \text { Kinematic Viscosity } \\ \rho_{f, m} & \text { Fluid Density Experimental Facility Model } \\ \rho_{f, P} & \text { Fluid Density Reactor Building Prototype } \\ \mathrm{Re} & \text { Reynolds Number } \\ S t k & \text { Stokes Number }\end{array}$




$\begin{array}{ll}\mathrm{U} & \text { Velocity } \\ \mu_{f, m} & \text { Fluid Viscosity of Experimental Facility Model } \\ \mu_{f, P} & \text { Fluid Viscosity of Reactor Building Prototype } \\ U_{f, m} & \text { Fluid Velocity of Experimental Facility Model } \\ U_{f, p} & \text { Fluid Velocity of Reactor Building Prototype }\end{array}$

\section{ACKNOWLEDGMENTS}

This work was performed under the U.S. Department of Energy Office of Nuclear Energy's Versatile Test Reactor program.

\section{References}

\section{REFERENCES}

[1] P. W. Humrickhouse, HTGR Dust Safety Issues and Needs for Research and Development, Idaho Falls: Idaho National Laboratory, 2011.

[2] J. Young and A. Leeming, "A theory of particle deposition in turbulent pipe flow," Journal of Fluid Mechanics, vol. 340, pp. 129-159, 1997.

[3] D. Orea, R. Chavez, D. T. Nguyen, R. Vaghetto, N. Anand, Y. Hassan and P. Sabharwall, "Experimental Investigation of Surrogate Particle Transport in a Turbulent Channel Flow Versatile Test Reactor Program," in ANS Winter Meeting 2019, 2019.

[4] S. R. Yang, E. Kappes, D. T. Nguyen, R. Vaghetto and Y. Hassan, "Experimental study on $1 / 28$ scaled NGNP HTGR reactor building test facility response to depressurization event," Annals of Nuclear Energy, vol. 114, pp. 154-164, 2018.

[5] C. A. Schneider, W. S. Rasband and K. W. Eliceiri, "NIH Image to ImageJ: 25 years of image analysis," Nature methods, vol. 9, no. 7, pp. 671-675, 2012.

[6] F. Fisher, A. Andris, W. Lippmann and A. Hurtado, "Particle Deposition by Thermophoresis Under High-Temperature Conditions in a Helium Flow," Journal of Nuclear Engineering and Radiation Science, vol. 4, 2018.

[7] B.-H. Choi, D. Orea, T. N. N. Anand, Y. Hassan and P. Sabharwall, "Numerical Investigation of Fluid Flow in a Square Channel - Versatile Test Reactor Program," in ANS Winter Meeting, 2019. 